Ann. Zootech., I970, 19 (I), I9-25.

\title{
EFFETS D'UNE SUBSTITUTION RÉCIPROQUE ENTRE LE SACCHAROSE ET L'AMIDON SUR LE POIDS DU JAUNE ET DU BLANC DE L'EUE ET SUR LA QUALITÉ DE LA COQUILLE
}

\author{
B. LECLERCQ, P. MONGIN et J.-C. BLUM \\ Station de Recherches avicoles, \\ Centre de Recherches de Tours, 37 - Nouzilly \\ Institut national de la Recherche agronomique
}

\section{RÉSUUMÉ}

La substitution à poids égal de saccharose à l'amidon de maîs dans un aliment pour poule pondeuse n'affecte ni l'intensité de ponte ni le poids de l'albumen. Seuls le poids du jaune et la solidité de la coquill s sont modifiés : l'un et l'autre augmentant sous l'action du saccharose. Si pour la coquille l'effet est lié à la présence de ce sucre dans le régime, il semble que le poids du jaune soit plus sensible à l'apport qu'au retrait du sucre.

\section{INTRODUCTION}

La nature et le mode de dégradation des glucides alimentaires ont une influence sur différents processus métaboliques : en particulier la biosynthèse et la mobilisation des lipides (ZADKIn et al., I967; COHEN et Tei'TELBAUM I968), l'absorption intestinale des minéraux (Fournier, I965; DAVENPORT I966; FordTraN et al., I968; BARRY I967). Plusieurs études sont consacrées à l'effet du saccharose sur la croissance du Poulet (PALAfox et Rosennberg I954; McGinnis ig67). Par contre, chez la Poule pondeuse nous n'avons connaissance que d'une seule expérience réalisée en I953 par RoSENBERG. Il en ressort que l'effet du saccharose sur l'intensité de ponte et le poids de l'œuf paraît faible et variable selon la dose. En fait les résultats obtenus peuvent être sujets à caution car les régimes utilisés ne sont pas tout à fait comparables; en particulier le sucre étant substitué à des céréales, l'apport alimentaire de protéines varie tant sur le plan qualitatif que quantitatif. L'intensité des 
métabolismes lipidique et minéral ainsi que l'intervention possible du saccharose dans ces métabolismes nous ont incité à effectuer une nouvelle étude dans laquelle une seule composante du régime varie : la nature de l'apport glucidique.

\section{MATÉRIEL E'T MÉTHODES}

Les poules, réparties en 3 lots de 24 animaux juste avant l'entrée en ponte, sont issues d'un croisement Rhode Island Red $\times$ Wyandotte. Elles sont élevées en cages individuelles, nourries et abreuvées à volonté et soumises à un éclairement journalier de 16 houres.

La composition des différents régimes complets et équilibrés fait l'objet du tableau I. Théoriquement, ils ne diffèrent que par la nature de l'apport glucidique : $5^{\circ} \mathrm{p}$. Ioo d'amidon (régime A) ; $5^{\circ} \mathrm{p}$. Ioo de saccharose (régime $\mathrm{S}$ ) ; $50 \mathrm{p}$. Ioo d'un mélange à parties égales saccharose-amidon (régime AS). Malheureusement le saccharose est dénaturé par de la farine de poisson $(2,5$ p. Ioo) et du chlorure de sodium (I. p. IOo). Nous tiendrons compte de ce fait dans la discussion. Les valeurs énergétiques du saccharose et de l'amidon étant très voisines (PotTER et MATTERson r960) les régimes peuvent être considérés comme isocaloriques.

TABLEAU I

Composition des régimes

\begin{tabular}{|c|c|c|c|}
\hline & Régine A & Régime S & Régime AS \\
\hline Gluten de maïs $\ldots \ldots \ldots \ldots \ldots \ldots \ldots \ldots \ldots \ldots \ldots$ & 15 & 15 & 15 \\
\hline Tourteau de soja $\ldots \ldots \ldots \ldots \ldots \ldots \ldots \ldots \ldots$ & 15 & 15 & 15 \\
\hline 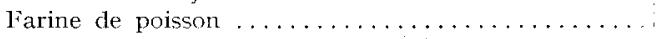 & 3 & 3 & 3 \\
\hline Huile de maïs $\ldots \ldots \ldots \ldots \ldots \ldots \ldots \ldots \ldots$ & 3 & 3 & 3 \\
\hline Farine de luzerne $\ldots \ldots \ldots \ldots \ldots \ldots \ldots \ldots \ldots$ & 5 & 5 & 5 \\
\hline Carbonate de calcium . . . . . . . . . . . . . & 7 & 7 & 7 \\
\hline Chlorure de sodium $\ldots \ldots \ldots \ldots \ldots \ldots \ldots \ldots$ & 0,2 & 0,2 & 0,2 \\
\hline Phosphate bicalcique ................. & 1,5 & 1,5 & 1,5 \\
\hline 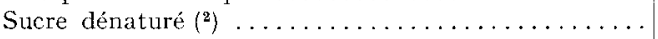 & 0 & 50 & 25 \\
\hline Amidon de mais $\ldots \ldots \ldots \ldots \ldots \ldots \ldots \ldots \ldots$ & 50 & 0 & 25 \\
\hline Mélange de minéral et vitaminique $\left.{ }^{1}\right) \ldots \ldots \ldots \ldots$ & 0,3 & 0,3 & 0,3 \\
\hline
\end{tabular}

(1) Composition du mélange minéral et vitaminique (pour $100 \mathrm{~kg}$ d'aliment) : vitamine A: 1000000 $\mathrm{UI}$; vitamine $\mathrm{D}_{3}: 100000 \mathrm{UI}$; riboflavine $: 0,5 \mathrm{~g}$; vitamine $\mathrm{E}: 8 \mathrm{~g}$, choline : $50 \mathrm{~g}$; $\mathrm{BHT}: 10 \mathrm{~g}$; DL-méthionine : $30 \mathrm{~g}$; sulfate de manganèse : $8 \mathrm{~g}$.

(2) Le sucre dénaturé contient 2,5 p. 100 de poisson et 1 p. 100 de chlorure de sodium.

L'expérience comprend deux périodes : la première dure 3 mois (période I), la seconde 6 semaines (période 2). Les régimes distribués au cours de ces deux périodes définissent chaque lot selon le tableau ci-dessous :
Lot $\mathrm{A} \rightarrow \mathrm{S} \ldots \ldots \ldots \ldots \ldots$
Lot $\mathrm{S} \rightarrow \mathrm{A} \ldots \ldots \ldots \ldots$
Lot $\mathrm{AS} \ldots \ldots \ldots \ldots$
Période 1
Régime A
Régime S
Régime AS
Pćriode 2
Régime $\mathrm{S}$
Régime A
Régime AS

L'intensité de ponte est contrôlée pendant toute la durée de l'expérience. A la fin de chacune de ces deux périodes, pendant les $\mathrm{I}_{4}$ derniers jours, on effectue les mesures suivantes sur tous les 
œufs pondus : poids total, poids du jaune débarrassé de l'albumen et essuyé sur papier-filtre, poids de la coquille après lavage et séchage à I0 $3^{\circ}$. On en déduit le poids de 1'albumen (cuf entier moins le jaune et la coquille). L'index de coquille est déterminé grâce à la formule :

$$
I=\frac{\text { Poids de la coquille } \times \text { Ioo }}{\text { Surface de la coquille }}
$$

la surface de l'œuf étant évaluée à l'aide de tables lorsque l'on connaît le poids de l'œuf entier (MoNGIN, I965).

Pour chaque critère et pour chaque poule, la moyenne de 5 valeurs de la période I sert de base de référence. Elle permet d'apprécier les effets des changements de régime en calculant les différences entre cette moyenne et 5 valeurs de la période 2 . L'analyse statistique porte sur ces différences. On peut ainsi dissocier l'effet propre du traitement et l'effet individuel. Par ailleurs les animaux sont classés en catégories sur la base du poids moyen des œufs pondus au cours de la période I : inférieurs à $60 \mathrm{~g}$, de 60 à $65 \mathrm{~g}$ et supérieurs à $65 \mathrm{~g}$. Ainsi on peut tenir compte d'un effet éventuel du poids de l'œuf et voir si le régime a une action différente sclon la grosscur de l'œuf.

\section{RÉSULTATS}

Nous avons tout d'abord constaté que l'ingestion de saccharose en quantité importante est très bien supportée par les poules ; aucun trouble digestif n'a été enregistré.

L'intensité de ponte n'est pas modifiée par les changements de régime. Elle reste de l'ordre de $70 \mathrm{p}$. Ioo pour les 3 lots et varie peu au cours de l'expérience.

Dans le tableat 2 nous indiquons les poids moyens de l'ouf, du jaune et du blanc ainsi que la valeur de l'index de coquille pour les deux périodes. Nous mentionnons également les résultats de l'analyse statistique.

Le poids de l'œuf entier augmente d'une période à l'autre dans tous les lots. Mais cette augmentation est significativement plus grande $(P<o$,oI $)$ dans le lot $\mathrm{A} \rightarrow \mathrm{S}$ que dans le lot $\mathrm{S} \rightarrow \mathrm{A}$. I'évolution du lot AS est intermédiaire. On peut faire les mêmes remarques à propos du jaune de l'œuf en notant toutefois que les lots AS et $S \rightarrow A$ ne diffèrent pas significativement. Ces augmentations de poids varient d'une poule à l'autre (effet poule hattement significatif), mais elles sont indépendantes de l'aptitude à pondre des œufs plus ou moins gros (effet taille de l'œuf non significatif). L'incorporation du sucre au régime a donc dans tous les cas un effet favorable sur l'augmentation du poids de l'œuf et du vitellus.

La quantité d'albumen contenue dans 1'ouf s'accroît, elle aussi, chez tous les animaux, mais cet accroissement est le même dans les 3 lots. Il n'est pas influencé par la nature de l'apport glucidique ; il dépend uniquement des aptitudes individuelles (effet poule).

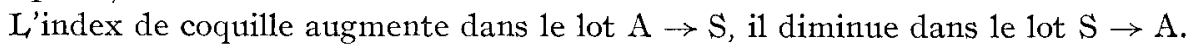
Une fois de plus le lot AS a un comportement intermédiaire; son index de coquille diminue très légèrement.

Sous l'effet de l'âge le poids du jatue et celtui de l'albumen varient normalement dans le sens de l'aungmentation ainsi que le montrent les résultats du lot AS. A l'opposé, la solidité de la coquille diminue $(-0,13$, lot $\mathrm{AS})$ quand la poule vieillit, si bien que pour le lot $\mathrm{A} \rightarrow \mathrm{S}$ l'effet propre du régime est une augmentation de $0,46(0,33+0, \mathrm{I} 3)$ et pour le lot $\mathrm{S} \rightarrow \mathrm{A}$ une diminution de $0,30(0,43-0, \mathrm{I} 3)$. Par ailleurs, quelle que soit la période considérée la solidité de la coquille est comparable chez les animaux qui reçoivent du saccharose; et cela indépendamment de la teneur du régime. 
B. LECLERCQ, P. MONGIN, J.-C. BLUM

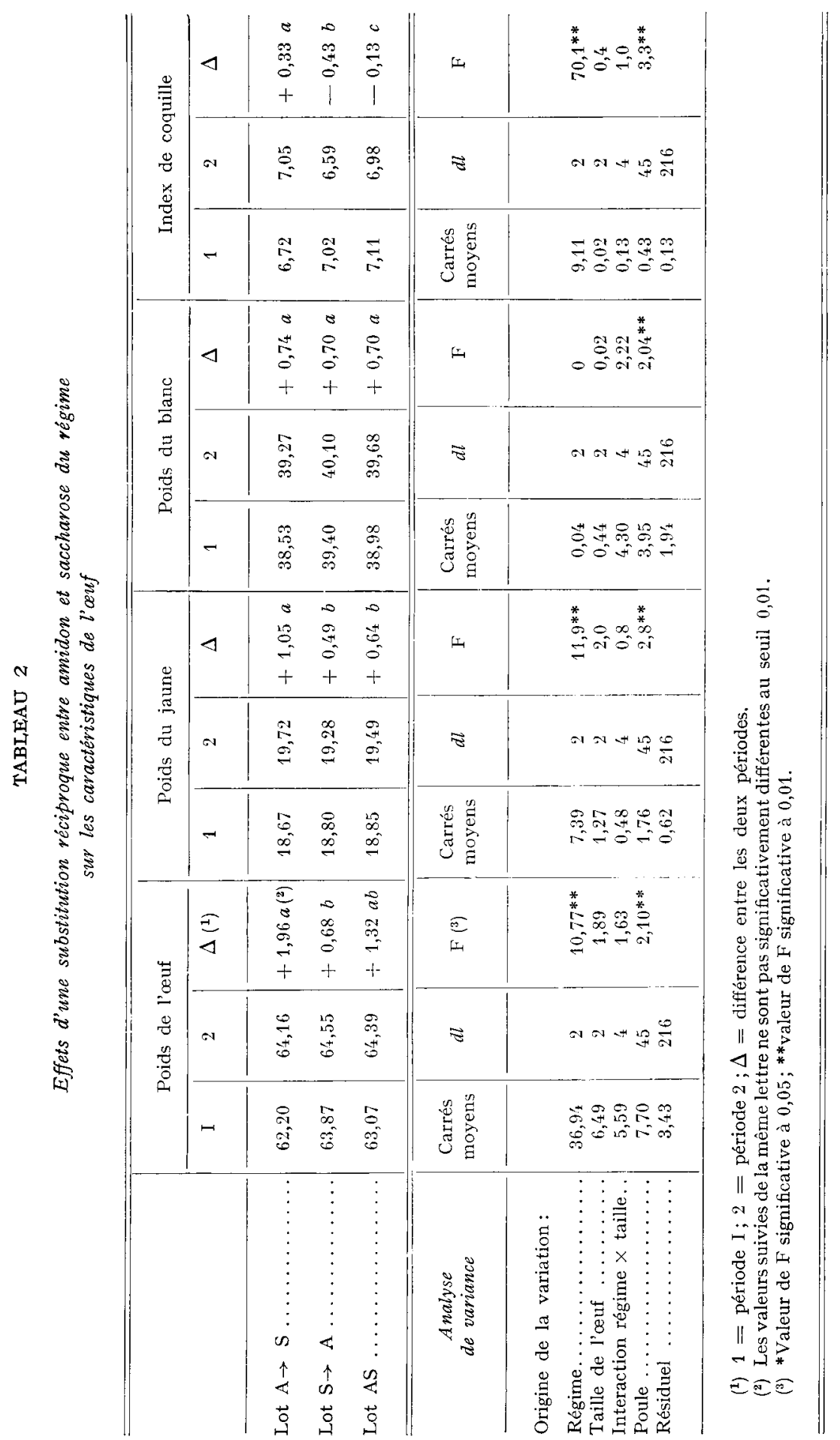




\section{DISCUSSION}

Chez les mammifères monogastriques, la substitution du saccharose à l'amidon augmente la lipogenèse (DESMOULIN, I967; ZADKIN et al., I967 ; CoHEN et TEITELBAUM, I 967) ; chez le Rat, la triglycéridémie s'élève (AKINYANYU et YuDKIN, I967). De telles modifications, si elles intéressent les oiseaux, pourraient expliquer l'accroissement de poids du jaune chez les poules ingérant du saccharose. Le remplacement de l'amidon par ce sucre aurait un effet stimulant sur la biosynthèse des lipoprotéines circulantes ; lipoprotéines incorporées dans le jaune en si forte quantité qu'elles forment la majeure partie de la matière sèche du vitellus. Si le métabolisme des lipides est seul modifié, on comprend que le poids de l'albumen (constituant dépourvu de lipides) soit indépendant de la nature des glucides alimentaires. Ainsi, ce comportement différent du jaune montre une fois de plus que 1'albuminogenèse et la vitello-

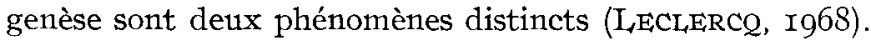

L'effet le plus spectaculaire concerne l'index de coquille. Alors que celui-ci tend à décroître avec l'âge et la taille de l'œuf, on constate que la substitution du sucre à l'amidon provoque une nette élévation de l'index. La suppression du sucre dans l'aliment ayant l'effet inverse. Du reste, indépendamment des changements de régime, au cours de chaque période on doit remarquer que les animaux disposant de sucre dans leur ration (régimes $\mathrm{S}$ et $\mathrm{AS}$ ) ont toujours un index de coquille significativement plus élevé $(P<0, O I)$ que ceux qui consomment exclusivement de l'amidon (régime $\mathrm{A}$ ). On pourrait assigner au saccharose un effet non spécifique propre à certains sucres simples appelés facteurs glucidiques d'utilisation du calcium (FourNIER, I965) et dont le lactose est le plus typique. Toutefois ces deux sucres diffèrent en ce qui concerne les processus d'absorption : influence sur le potentiel de membrane (BARRY, I967), hydrolyse dans la bordure en brosse (DAVENPORT, I966). L'absorption intestinale du calcium est sous la dépendance d'un transfert actif qui utilise les phosphates à pouvoir énergétique élevé produit par le métabolisme aérobie (DAVENPORT, I966). On peut se demander alors si le saccharose après son hydrolyse par la bordure en brosse n'a pas un effet sur la production intracellulaire d'énergie utilisable pour le transfert du calcium. En outre, on peut se demander si le transport actif du calcium n'est pas facilité par les sucres simples selon un mécanisme de couplage déjà démontré dans le cas du sodium (Fordtran et al., rg68). Infin il reste la possibilité d'actions métaboliques au niveau du foie ou de l'utérus dues au devenir particulier des produits d'hydrolyse du saccharose dans l'organisme.

Pour terminer il nous faut évoquer la présence de farine de poisson et de chlorure de sodium dans le sucre dénaturé. Leur intervention est peu probable car ils figurent déjà dans la ration en quantité importante au moins pour la farine de poisson. En outre, ce sont des matières premières dont les effets sur la qualité et la composition de l'œuf ont fait l'objet de nombreuses études. Le poisson apportant surtout des protéines devrait influencer la sécrétion de l'albumen. Or, il n'en est rien. Par ailleurs le chlorure de sodium rend les coquilles plus fragiles. Autant de faits qui rendent encore plus manifeste l'action du sucre.

En conclusion nos résultats posent de nouveau le problème des matières pre- 
mières utilisées comme source d'énergie dans l'alimentation des volailles mais surtout de leur dégradation et de leur absorption. Quelques études ont déjà montré l'importance de ces phénomènes chez l'Oiseau (IVOREC-Szylit et al., I965 ; IvorecSzyirit et SzyLIT, I965). De plus sur le plan zootechnique nous démontrons 1'effet bénéfique du saccharose à la fois sur la taille du jaune de l'œuf et sur la solidité de la coquille. Cette stimulation qui n'est peut-être que temporaire dans le premier cas semble durable et liée uniquement à l'ingestion de saccharose dans le second. D'autres essais doivent être réalisés afin de préciser l'effet de la dose et de l'époque de ponte la plus propice. Nous essaierons également d'utiliser d'autres oses ou diholosides en cherchant à préciser les mécanismes responsables. En définitive l'usage du saccharose dans la ration pourrait être intéressant si la solidité de la coquille était accrue par un faible apport alimentaire. La valeur de l'index paraît être identique que le régime contienne 25 ou $50 \mathrm{p}$. Ioo de sucre; une plus faible dose aurait peut-être encore la même influence.

Rę̧u pour publication en mars $19 \% 0$.

\title{
SUMMARY
}

\author{
EFFECT OF RECIPROCAL SUBSTITUTION BETWEEN SUGAR AND STARCH \\ UPON THE YOLK AND WHITE WEIGHT OF THE EGG \\ AND UPON THE QUALITY OF THE SHEI,
}

Reciprocal substitution of starch by sugar and of sugar was studied in the laying-hen. When changing a diet without sugar into a diet containing 50 per cent saccharose, the increase of the weight of the egg and the yolk is higher than that obtained by an inverse change or by maintening the diet at a level of 25 per cent and 25 per cent starch. Moreover, the resistance of the shell is always much higher when the diet contains saccharose.

\section{RÉFÉRENCES BIBLIOGRAPHIQUES}

Akinyanju P. A., Yudrin J., 1967. Effects of dietary carbohydrates on the serum lipids of the Rat. Proc. Nutr. Soc. England-Scotland, 26, XXXi.

BARRY R. J. C., 1967. Electrical changes in relation to transport. Br. Med. Bull, 23, 266-269.

Comen A. M., Teitelbaum L., I968. Effect of glucose, sucrose, fructose and starch on lipogenesis in rats. Life Sci., 7, 20-30.

Davenport H. W., I966. Physiology of digestion tract. Year Book medical publisher Inc. Chicago, Ill.

Desmoulin $B$. Influence de l'amidon de maïs et du saccharose sur la consommation des régimes selon le taux azoté : conséquences sur la composition corporelle du Rat blanc. Ann. Biol. anim. Bioch. Biophvs., $7, \mathbf{1} 63-172$.

Fordtran J. S., Rector F. C., Carter N. W., I968. The mechanism of sodium absorption in the human small intestine. J. Clin. Invest., 4\%, 884-9oo.

Fournier P., I965. Influence du lactose sur l'utilisation du calcium et sur l'ossification. Rev. Path. Biol., 12, I43-I5I.

Ivorec-Szylit O., Mercier C., Raibaud P., Calet C., i965. Contribution à l'étude de la dégradation des glucides dans le jabot du coq. Influence du taux de glucose dans le régime sur l'utilisation de l'amidon. C. R, Acad., Sci., 261, 3201-3203.

Ivorec-Szylit O., Szylit M., I965. Contribution à l'étude de la dégradation des glucides dans le jabot du coq. Mise en évidence et dosage des stéréoisomères D- et L-lactate. Ann. Biol. anim. Bioch. Biophys., 5, $353-360$.

LeclercQ B., I968. Facteurs nutritionnels modifiant indépendamment le poids de l'œuf et de ses constituants. $3^{e}$ Congrès Européen d'Aviculture. Jérusalem. 
Mongin P., I965. Index de solidité de la coquille de l'œuf. Ses significations. Sa précision. Ann. Zootech., 14, 3I9-325.

McGinNis J., I967. Relationship of dietary amino acids to the other nutrients components of poultry diets. in Protein utilization by poultry. Oliver et Boyd Edinburg p. I7r.

Palafox A. L., Rosenberg M. M., I954. An evaluation of low grade sugar in starter and grower rations of chickens. Poult. Sci., 33, I27-I33.

Potter L. M., Matterson L. D., I960. Metabolisable energy of feed ingredients for the growing chick.

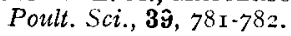

Rosenbers M. M., I953. Low grade sugar a potential carbohydrate feedstuff for laying chickens. Poult. Sci, 32, 69-77.

Zadiin D., Pardini R S., Herman R. H., Sauberlich H. E., ig67. Mechanism for the differential effect of high carbohydrate diets on lipogenesis in rat liver. Bioch. Biophys. Acta., 144, 242-25 I. 